\title{
A Novel Sit4 Phosphatase Complex Is Involved in the Response to Ceramide Stress in Yeast
}

\author{
Alexandra Woodacre, ${ }^{1}$ Museer A. Lone, ${ }^{2}$ Daniel Jablonowski, ${ }^{1,3}$ Roger Schneiter, ${ }^{2}$ \\ Flaviano Giorgini, ${ }^{1}$ and Raffael Schaffrath ${ }^{1,3}$ \\ ${ }^{1}$ Department of Genetics, University of Leicester, Leicester, LE1 7RH, UK \\ ${ }^{2}$ Division of Biochemistry, Department of Biology, University of Fribourg, CH-1700 Fribourg, Switzerland \\ ${ }^{3}$ Institut für Biologie, FG Mikrobiologie, Universität Kassel, 34132 Kassel, Germany \\ Correspondence should be addressed to Flaviano Giorgini; fg36@le.ac.uk and Raffael Schaffrath; schaffrath@uni-kassel.de
}

Received 10 May 2013; Revised 28 June 2013; Accepted 25 July 2013

Academic Editor: Joris Winderickx

Copyright (C) 2013 Alexandra Woodacre et al. This is an open access article distributed under the Creative Commons Attribution License, which permits unrestricted use, distribution, and reproduction in any medium, provided the original work is properly cited.

Ceramide is a building block for complex sphingolipids in the plasma membrane, but it also plays a significant role in secondary signalling pathways regulating cell proliferation and apoptosis in response to stress. Ceramide activated protein phosphatase activity has been previously observed in association with the Sit4 protein phosphatase. Here we find that sit $4 \Delta$ mutants have decreased ceramide levels and display resistance to exogenous ceramides and phytosphingosine. Mutants lacking SIT4 or KTI12 display a shift towards nonhydroxylated forms of long chain bases and sphingolipids, suggesting regulation of hydroxylase (SUR2) or ceramide synthase by Sit4p. We have identified novel subunits of the Sit 4 complex and have also shown that known Sit 4 regulatory subunitsSAP proteins-are not involved in the ceramide response. This is the first observation of separation of function between Sit 4 and SAP proteins. We also find that the Sit $4 p$ target Elongator is not involved in the ceramide response but that cells deficient in Ktil2pan accessory protein with an undefined regulatory role-have similar ceramide phenotypes to sit $4 \Delta$ mutants. Therefore, Ktil2p may play a similar secondary role in the ceramide response. This evidence points to a novel Sit4-dependent regulatory mechanism in response to ceramide stress.

\section{Introduction}

Ceramide is a building block for complex sphingolipids which comprise an important structural component of the plasma membrane. It is also a secondary signalling molecule that accumulates in response to stresses such as heat shock [1]. It is therefore important for sphingolipid metabolism to be tightly regulated, and the damaging effects of dysregulation are apparent in patients with Tay-Sachs disease, Fabry disease, and other inherited sphingolipidosis disorders [2]. Ceramide mediates controlled cell death by triggering several signalling cascades to initiate caspase-dependent and independent apoptosis [3]. In contrast, the phosphorylated ceramide precursors dihydrosphingosine (DHSP) and phytosphingosine (PHSP) are signals for pathways that promote cell proliferation $[4]$.
Although it is known that the cellular response to ceramide is important for the regulation of cell proliferation and cell death pathways, the precise molecular mechanisms for this regulation still remain elusive. It is vital to further understand the way cells respond to stress in order to develop strategies to modify them, either to accelerate cell death using targeted anticancer drugs or to prevent accumulation of toxic products in sphingolipidoses [5-7].

Saccharomyces cerevisiae has been used effectively as a model to study sphingolipid metabolism, and Figure 1 shows a detailed summary of the sphingolipid biosynthetic pathway in yeast [8]. Many of the genes involved are conserved from yeast to higher eukaryotes, with diversion in the synthesis of complex sphingolipids occurring only after the production of ceramides, resulting in the production of different end products in the pathway. The addition of inositol to ceramide in 


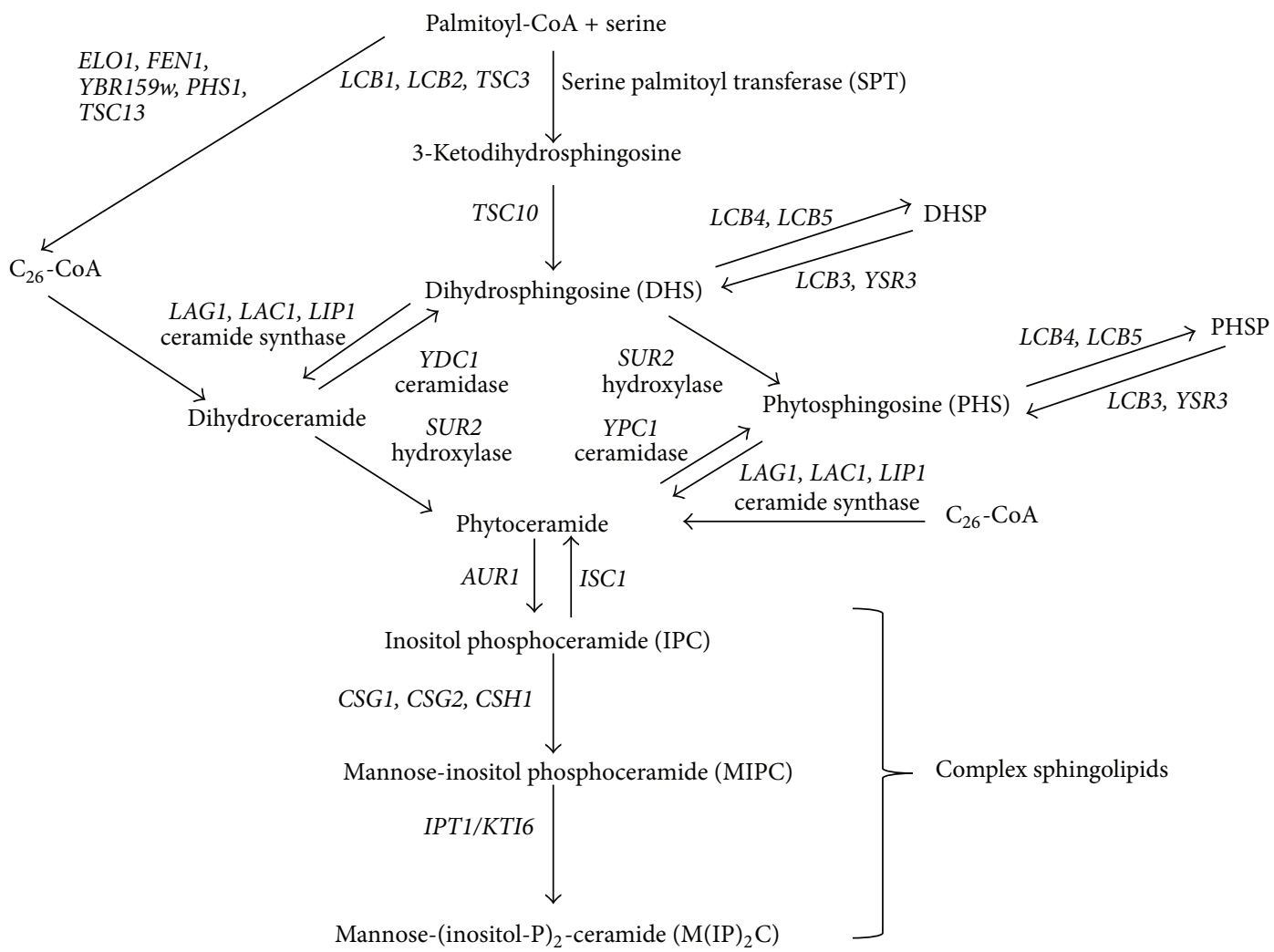

FIGURE 1: Biosynthesis of sphingolipids in Saccharomyces cerevisiae. Key enzymes discussed in the text are highlighted and genes encoding all relevant parts of the pathway are included. The directions of arrows indicate the end products of enzymatic reactions.

yeast forms inositol phosphoceramide, and glucose, galactose or phosphorylcholine is added to ceramide in mammalian cells to generate glycosphingolipids and sphingomyelin [9].

Early work by Nickels and Broach showed that a ceramide-activated phosphatase activity was present in Saccharomyces cerevisiae, which is separate from the activity of the major PP2A phosphatases Pph21p and Pph22p [10]. The ceramide resistance of a sit $4 \Delta$ mutant strain suggested that the PP2A-like phosphatase Sit4p is responsible for this activity. SIT4 is an essential gene in the absence of the suppressor allele SSD1- $v$ and has important roles in the progression of the cell cycle, cell integrity, nutrient responses via TORC1, drug resistance via efflux pumps, and tRNA modification [11-16]. Diverse regulatory subunits of Sit4p are partially responsible for the different specificities of Sit4p; for example, Tap42p is phosphorylated by Tor and binds Sit4p [17] and Sap185p and Sap190p subunits are essential for correct phosphoregulation of Elongator and tRNA modification [18]. Mutation of the SAPs (Sit4 associated proteins) can confer different specificities on Sit4p but a deletion of all four SAPs always results in the same phenotypes as deletion of SIT4, for example resistance to the tRNAse toxin zymocin, cell cycle arrest, and sensitivity to rapamycin $[19,20]$. The accessory protein Kti12p is also essential for the phosphoregulation of the Elongator subunit Elp1p by the Sit4p/Sap185p/Sap190p complex. Kti12p interacts with the casein kinase Hrr25p in an Elongator-dependent manner but the mechanism by which Kti12p regulates phosphorylation remains unclear [21].

The aim of this study was to further investigate the role of Sit4 as the ceramide-activated protein phosphatase (CAPP) in yeast. We identify KTI12 as an important gene mediating ceramide toxicity and show that ceramide toxicity is independent of Elongator function. Mutants lacking SIT4 or KTI12 have decreased levels of ceramide and the balance of hydroxylated and nonhydroxylated sphingolipids is altered. The confirmation that Tpd3p and Cdc55p can interact with Sit4p and a separation of function between SIT4 and the regulatory SAP subunits indicates that the CAPP is likely to be an alternative Sit 4 complex operating via a novel mechanism.

\section{Methods}

2.1. Yeast Strains and Media. Yeast were routinely grown in yeast extract peptone dextrose medium (YPD; $1 \%$ yeast extract, $1 \%$ peptone, $2 \%$ glucose) at $30^{\circ} \mathrm{C}$ with shaking. Glucose was replaced with $2 \%$ galactose to induce expression of SIT4 and PPH21 from the GAL1 promoter. Synthetic defined medium without inositol $(0.67 \%$ yeast nitrogen base, $2 \%$ glucose, supplemented with essential amino acids) was used for labelling with $\left[{ }^{3} \mathrm{H}\right]$ myo-inositol. Yeast strains used in this study are listed in Table 1 . 
TABLE 1: Yeast strains used in this study.

\begin{tabular}{|c|c|c|}
\hline Strain & Genotype & Reference \\
\hline CY4029 & Mat a ade2-1 his3-11,15 leu2-3,112 trp1-1 ura3-1 can1-100 SSD1-v1 gal+ & {$[20]$} \\
\hline CY3938 & CY4029, sit4A::HIS3 & {$[20]$} \\
\hline CY5236 & 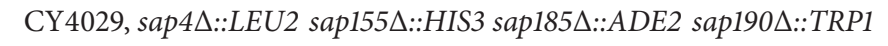 & {$[20]$} \\
\hline CY5220 & 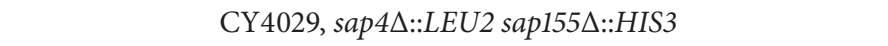 & {$[20]$} \\
\hline CY5224 & 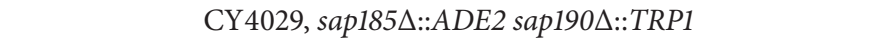 & {$[20]$} \\
\hline CY4917 & CY4029, sap185 $::$ ADE2 & {$[20]$} \\
\hline CY4380 & CY4029, sap190A::TRP1 & {$[20]$} \\
\hline DJY101 & 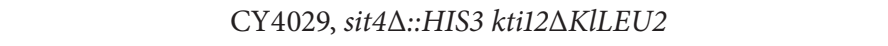 & {$[18]$} \\
\hline LFY3 & Mat a ade2-1 his3-11,15 leu2-3,112, ura3-1 can1-100, elp1D::TRP1 & {$[18]$} \\
\hline LFY4 & Mat a ade2-1 his3-11,15 leu2-3,112, ura3-1 can1-100, elp2 & {$[18]$} \\
\hline LFY5 & Mat a ade2-1 his3-11,15 leu2-3,112, ura3-1 can1-100, elp3D::TRP1 & {$[22]$} \\
\hline LFY6 & Mat a ade2-1 his3-11,15 leu2-3,112, ura3-1 can1-100, kti12A::TRP1 & {$[22]$} \\
\hline AWY1 & CY4029 TRP1::GAL1::(HA) $)_{3}$-SIT4 CDC55-(c-myc) $:: H I S 3 M X 6$ & This study \\
\hline AWY2 & CY4029, kanMX6::PGAL1::(HA) $)_{3}-P P H 21$, CDC55-(c-myc) $3:: H I S 3 M X 6$ & This study \\
\hline AWY3 & CY4029, CDC55-(c-myc) ${ }_{3}:: H I S 3 M X 6$ & This study \\
\hline
\end{tabular}

2.2. Growth Tests Using Ceramide and Long Chain Bases. C2ceramide, C2-phytoceramide, dihydrosphingosine (DHS), and phytosphingosine (PHS) powders were purchased from Enzo Life Sciences and resuspended in 100\% ethanol. Stock solutions $(5 \mathrm{mg} / \mathrm{mL})$ were stored at $-20^{\circ} \mathrm{C}$ for a maximum of 1 week. Yeast cultures were diluted from a starter culture to $5 \times 10^{3}$ cells $/ \mathrm{mL}$ in YPD containing ceramides/long chain bases or an equal volume of ethanol as an untreated control. Cultures were grown until the untreated control reached exponential phase (from 15-36 hours depending on the strain) and the $\mathrm{OD}_{600}$ measured for both treated and untreated cultures. After 24 hours, an additional dose of ceramide/long chain base was added to counteract the effects of compound degradation. The amount of growth in each concentration of ceramide/long chain base was then expressed as a percentage of the growth in untreated media. This method of standardising growth enables the comparison of slow-growing mutants such as sit $4 \Delta$ to a fastergrowing wild-type strain. Raw $\mathrm{OD}_{600}$ data is provided in Supplementary Tables 1 and 2 (see Supplementary Material available online at http://dx.doi.org/10.1155/2013/129645). A minimum of three biological replicates were performed for each strain and a one-way ANOVA with Bonferroni post-test was used to determine if growth was significantly different from the wild type (CY4029).

2.3. Immunoprecipitation. Dynabeads (Invitrogen) were coupled with $5 \mu \mathrm{g}$ of anti-HA antibody per mg of beads, following the manufacturer's instructions. Total protein extracts were prepared from $50 \mathrm{~mL}$ cultures grown for 8 hours in YPD supplemented with galactose. Cell pellets were resuspended in $400 \mu \mathrm{L}$ B60 buffer (50 mM HEPES pH 7.3, $60 \mathrm{mM}$ sodium acetate, $5 \mathrm{mM}$ magnesium acetate, $0.1 \%$ Triton $\mathrm{X}-100,10 \%$ glycerol, $1 \mathrm{mM}$ sodium fluoride, $20 \mathrm{mM}$ glycerophosphate, 1 mM DTT, 1X Complete Mini Protease Inhibitor Cocktail (Roche)). An equal volume of glass beads was added and cells disrupted using a bead beater for 1 minute, followed by centrifugation at $15700 \mathrm{~g}, 4^{\circ} \mathrm{C}$ for 5 minutes. The supernatant was transferred to a new tube and centrifuged at $15700 \mathrm{~g}, 4^{\circ} \mathrm{C}$ for 20 minutes. The cleared protein extract was quantified using spectrophotometry and $3.5 \mathrm{mg}$ of total protein extract was incubated with $1.5 \mathrm{mg}$ of antibody-coated beads for 30 minutes at $4^{\circ} \mathrm{C}$. Unbound proteins were removed by three washes with $1 \mathrm{~mL}$ B60 buffer, and antibody-bound proteins were eluted with $50 \mu \mathrm{L}$ of $10 \%(\mathrm{v} / \mathrm{v})$ SDS for 10 minutes at room temperature. The beads were then removed with a magnet and the supernatant used for Western blot analysis. SDS-PAGE of $100 \mu \mathrm{g}$ of total protein from each strain and immunoprecipitation supernatants (equal volumes) was carried out using $12 \%$ acrylamide gels and then Western blotted at $100 \mathrm{~V}$ for 1 hour. Blots were probed with anti-HA (F7 Santa Cruz), anti-c-myc (A14 Santa Cruz), or anti-Tpd3 (Y. Jiang, University of Pittsburg School of Medicine, USA) and secondary antibodies conjugated to horseradish peroxidase (Roche Diagnostics) were detected by chemiluminescence and exposed to X-ray film.

2.4. Sphingolipid Analysis by ESI-MS. Overnight cultures grown at $24^{\circ} \mathrm{C}$ in YPD media were diluted to $\mathrm{OD}_{600} 0.2$ and grown until they reached $\mathrm{OD}_{600}$ of 2 . A total of $10 \mathrm{OD}$ units of cells were collected and washed once with sterile water. Lipid extraction was performed by a two-step lipid extraction method [23]. Cells were resuspended in $1 \mathrm{~mL}$ of $150 \mathrm{mM}$ ammonium bicarbonate $\left(\mathrm{NH}_{4} \mathrm{HCO}_{3}\right)$ and $600 \mu \mathrm{L}$ of glass beads were added. After cell lysis using a Precellys 24 homogenizer ((Bertin technologies) $5000 \mathrm{rpm}, 3 \mathrm{x} 30 \mathrm{sec}$ on $30 \mathrm{sec}$ off), lysates were diluted in $5 \mathrm{~mL}$ of $150 \mathrm{mM}$ $\mathrm{NH}_{4} \mathrm{HCO}_{3}$ solution and internal standards were added. Long chain bases and ceramides were quantified relative to respective lipid standards, and inositol phosphoceramides were measured relative to a phosphoinositol standard. Lipid standards were purchased from Avanti Polar Lipids. ESI-MS analysis was performed using a Bruker Esquire HCT ion trap mass spectrometer in positive or negative ion mode. Peaks 


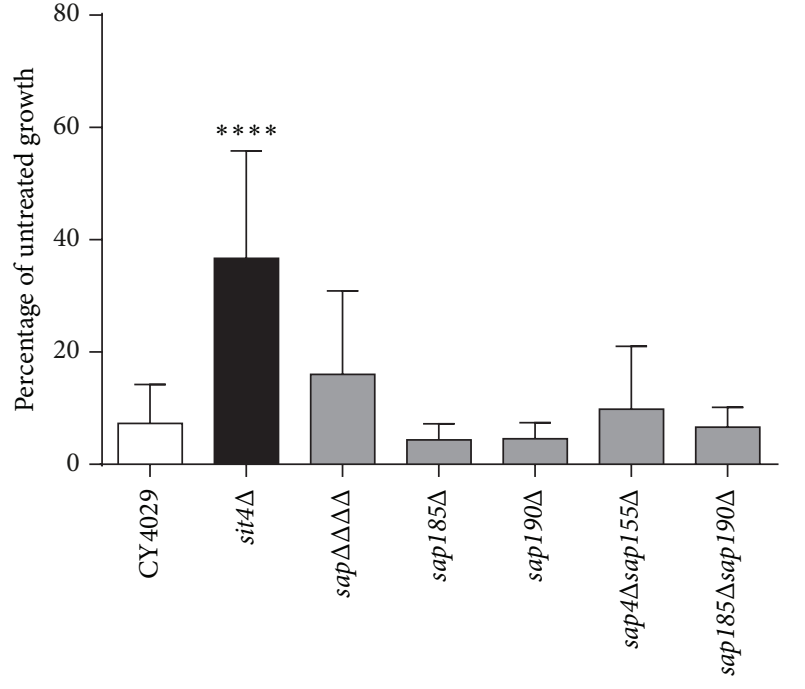

(a)

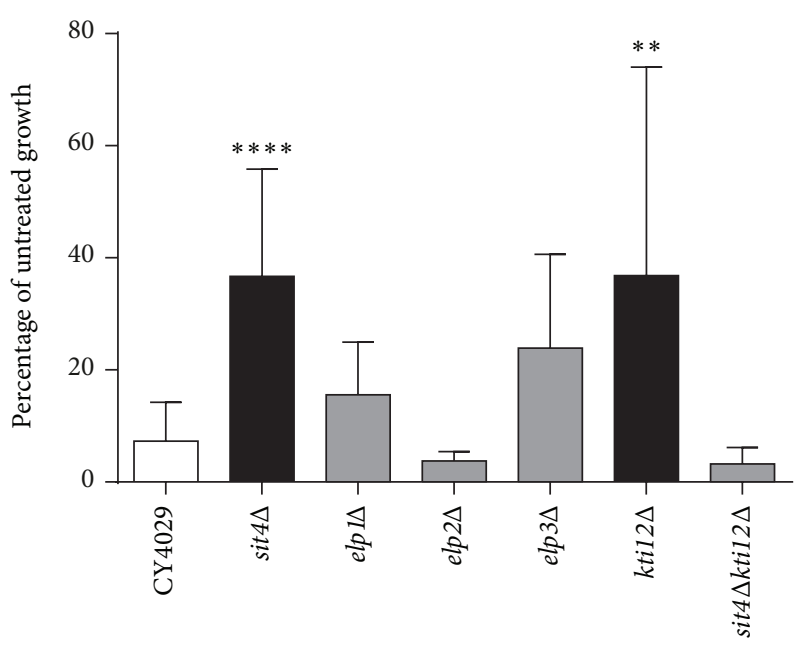

(b)

FIGURE 2: Deletion of SIT4 or KTI12 confers resistance to excess dihydroceramide. (a) Ceramide growth tests in Sit4 associated protein (SAP) mutants. (b) Ceramide growth tests in Elongator-associated mutants. Yeast cultures were diluted to $5 \times 10^{3}$ cells $/ \mathrm{mL}$ in YPD with the addition of either $15 \mu \mathrm{M}$ C-2 dihydroceramide or an equal volume of ethanol. Cells were grown until the untreated culture reached exponential phase and the $\mathrm{OD}_{600}$ of all cultures was determined. Growth in $15 \mu \mathrm{M}$ dihydroceramide is expressed as a percentage of untreated growth. Raw $\mathrm{OD}_{600}$ values are given in Supplementary Table 1. A minimum of three replicates are shown and error bars represent the standard deviation above and below the mean. A one-way ANOVA with a Bonferroni post-test was used to determine if mutants showed a significant difference in growth compared to the wild type (CY4029) $\left({ }^{* *} P<0.01,{ }^{* * *} P<0.001\right.$, and $\left.{ }^{* * * *} P<0.0001\right)$.

were identified based on their fragmentation pattern and by comparison to commercially available standards. Three biological replicates were included in each analysis.

2.5. Incorporation of $\left[{ }^{3} \mathrm{H}\right]$-Labelled Inositol. Overnight cultures grown at $24^{\circ} \mathrm{C}$ in YPD were diluted to $\mathrm{OD}_{600} 1.0$ in synthetic defined media containing $40 \mu \mathrm{Ci}\left[{ }^{3} \mathrm{H}\right]$ myo-inositol (American Radiolabelled Chemicals, MO, USA) and grown at $24^{\circ} \mathrm{C}$ for $4 \mathrm{~h}$ until they reached $\mathrm{OD}_{600}$ of approximately 2 . A total of $10 \mathrm{OD}$ units were harvested, and lipids were extracted using chloroform/methanol/water $(10: 10: 3)$ and analysed as previously described [24] by thin layer chromatography with or without mild-base treatment. Mild-base treatment to remove inositol phosphate and leave only $\mathrm{N}$-acetylated sphingolipids was performed by incubating lipids in $0.1 \mathrm{M}$ $\mathrm{NaOH}$ at $30^{\circ} \mathrm{C}$ for 1 hour. Radioactivity was detected using a phosphorimager (Typhoon FLA9500, GE Healthcare) and a representative image of two biological replicates is shown.

\section{Results}

3.1. Deletion of Sit4-Associated Proteins (SAPs) Does Not Confer Resistance to Exogenous Dihydroceramide. As previously described [10], deletion of SIT4 leads to significant resistance to $15 \mu \mathrm{M}$ dihydroceramide (Figure $2, P<0.0001$ ). However, mutation of the four SAP regulatory proteins, either individually or in combination, does not confer resistance to dihydroceramide (Figure 2(a)). In previous studies, the phenotype of the quadruple sap mutant has been indistinguishable from that of sit4 $\Delta[19,20]$. Thus, the ceramide sensitivity of the sap mutant is the first observed separation of function between $\operatorname{sit} 4 \Delta$ and $\operatorname{sap} \Delta \Delta \Delta \Delta$.

\subsection{Kti12p Appears to Be the Only Elongator-Associated} Protein Involved in the Ceramide Response. As Sit4p plays a major role in the phosphoregulation of the Elongator complex $[18,21]$ and previous studies suggested that Elongator mutants were resistant to ceramide, Elongator components were investigated as potential targets of Sit $4 p$ in the response to excess dihydroceramide. Although deletion of Elongator subunits did not confer statistically significant resistance to $15 \mu \mathrm{M}$ dihydroceramide, deletion of the Elongator accessory protein Ktil2p did confer resistance (Figure 2(b)) to some extent, though the obtained data were rather variable (Supplementary Table 1). Interestingly, deletion of SIT4 and KTI12 in tandem restored sensitivity to dihydroceramide, whereas in a previous study mutants lacking one or both of these genes had the same phenotype that resulted in hyperphosphorylation of Elplp and zymocin resistance [18]. In addition, phosphorylation of Elplp was unchanged in the presence of dihydroceramide, and this was not affected by deletion of SIT4 and/or KTI12 (data not shown). Therefore, our data suggest that Kti12p might play a regulatory role in the ceramide response that is independent of Elongator.

3.3. PHS Resistance of sit4D Indicates Separation of Function from kti12 $\Delta$. Growth in phytoceramide decreases in a concentration-dependent manner in both wild-type and mutant strains; however, sit4 $\Delta$ and kti12 $\Delta$ mutants show significantly more growth $(P<0.005)$ than the parental 


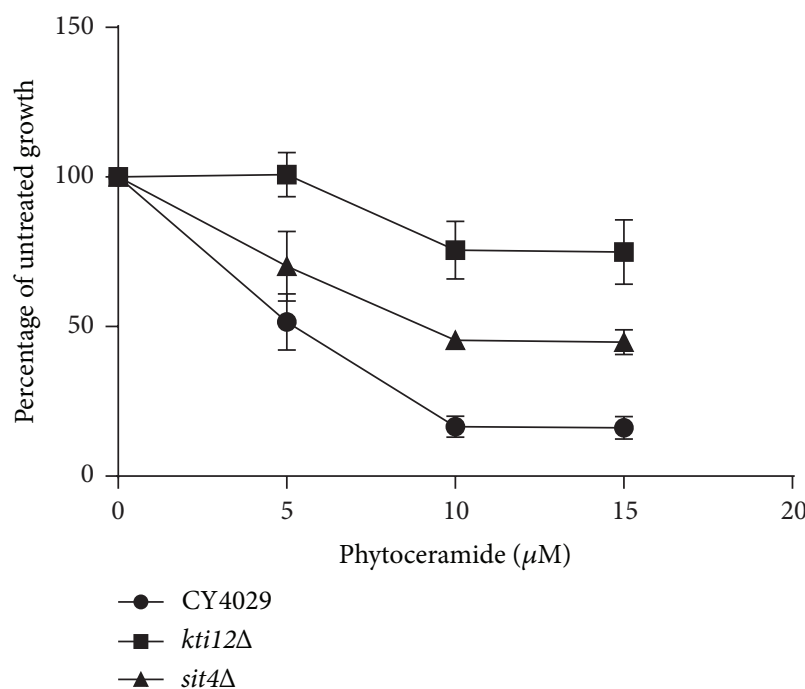

(a)

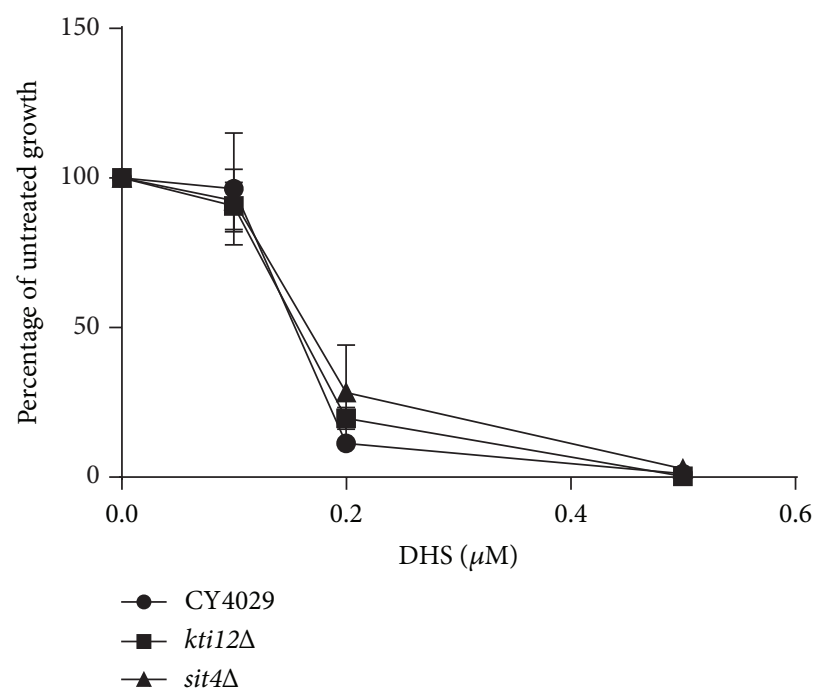

(b)

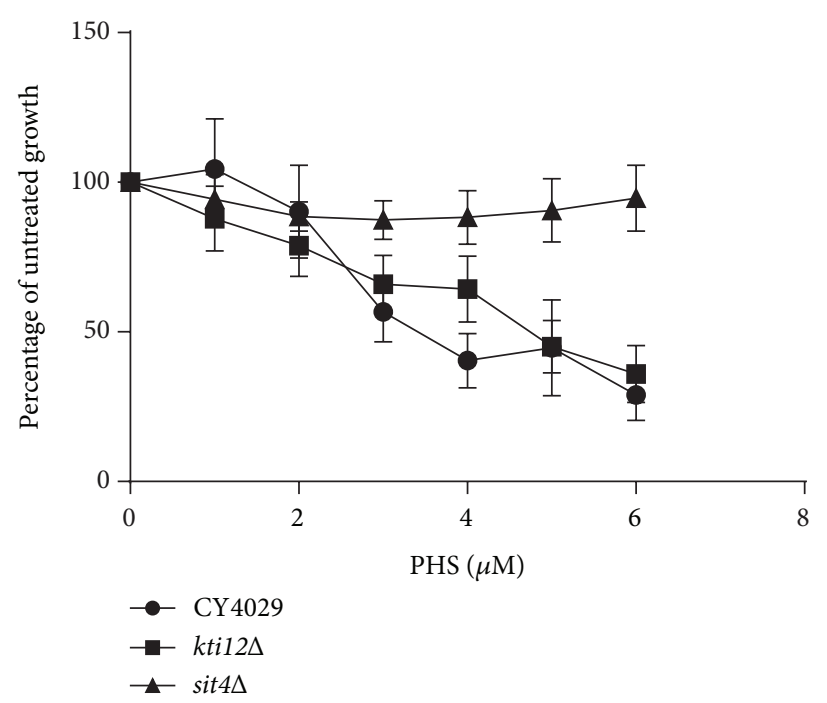

(c)

FIGURE 3: Response of sit4 $\Delta$ and kti12 $\Delta$ mutants to phytoceramide and long chain bases. Yeast cultures were diluted to $5 \times 10^{3}$ cells/mL in YPD with the addition of the indicated concentrations of (a) phytoceramide, (b) dihydrosphingosine (DHS), (c) phytosphingosine (PHS), or an equal volume of ethanol. Cells were grown until the untreated culture reached exponential phase and then the OD ${ }_{600}$ of both treated and untreated cells was measured and plotted as a percentage of untreated growth. Raw $\mathrm{OD}_{600}$ values are given in Supplementary Table 2. A minimum of three replicates are shown and error bars represent the standard error above and below the mean. A Student's $t$-test was used to determine if the mutants showed a significant difference in growth compared to the wild type (CY4029) at each concentration shown.

CY4029 strain at concentrations of $10-15 \mu \mathrm{M}$ (Figure 3(a)). In contrast, growth of sit4 $\Delta$ and $k t i 12 \Delta$ mutants in excess dihydrosphingosine (DHS) is indistinguishable from CY4029 (Figure 3(b)). The most striking result is that while $k t i 12 \Delta$ is also sensitive to phytosphingosine (PHS), sit4 $\Delta$ shows significant $(P<0.05)$ resistance to 3-6 $\mu \mathrm{M}$ PHS (Figure 3(c)), suggesting divergence of function between Ktil2p and Sit $4 \mathrm{p}$ in the response to long chain bases.

3.4. Ceramide and Long Chain Base Levels Are Reduced in sit $4 \Delta$ and kti12 $\Delta$ Mutants. To investigate the possibility that Sit4p and Kti12p regulate the sphingolipid biosynthesis pathway, we measured steady state levels of ceramides, long chain bases, and inositol phosphate in sit4 $\Delta$ and $k t i 12 \Delta$ strains relative to wild-type yeast cells. Deletion of SIT4 or KTI12 reduces the intracellular levels of phytoceramide by approximately 50\% (Figure 4(a)). Levels of dihydroceramide are also reduced in both mutants, but the decrease is only statistically significant in sit4 $\Delta$ (Figure $4(\mathrm{a})$ ). This suggests that the mutants may be able to tolerate otherwise toxic levels of exogenous ceramides due to the constitutively lower levels present within the cell. The reduction of PHS levels by approximately two-thirds in the sit $4 \Delta$ mutant could permit the strain to survive excess concentrations of PHS 


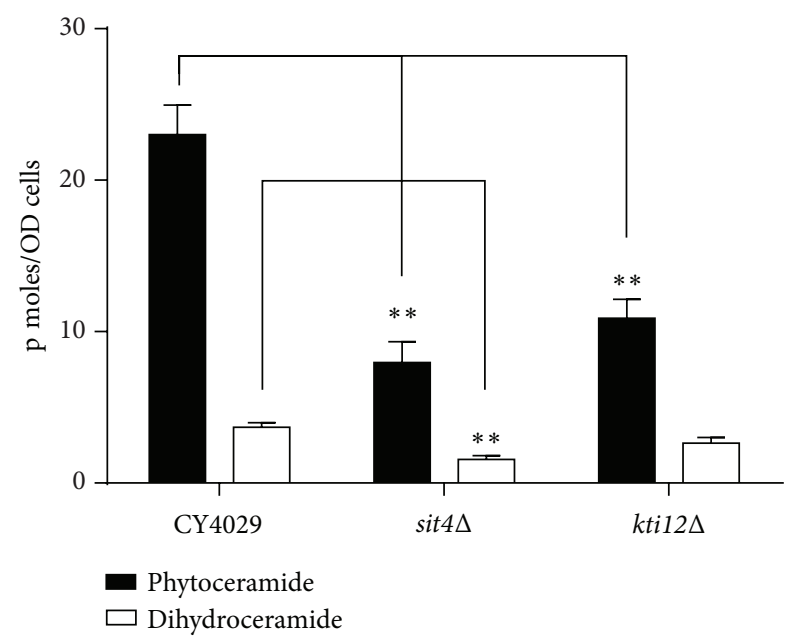

(a)

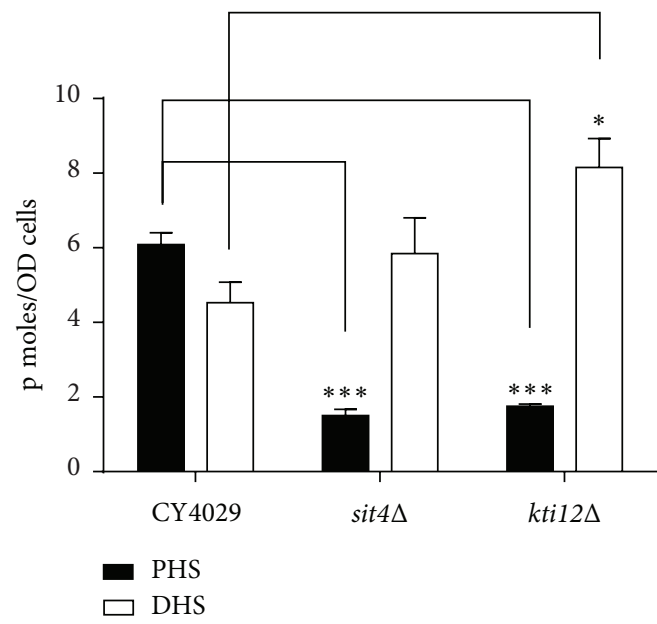

(b)

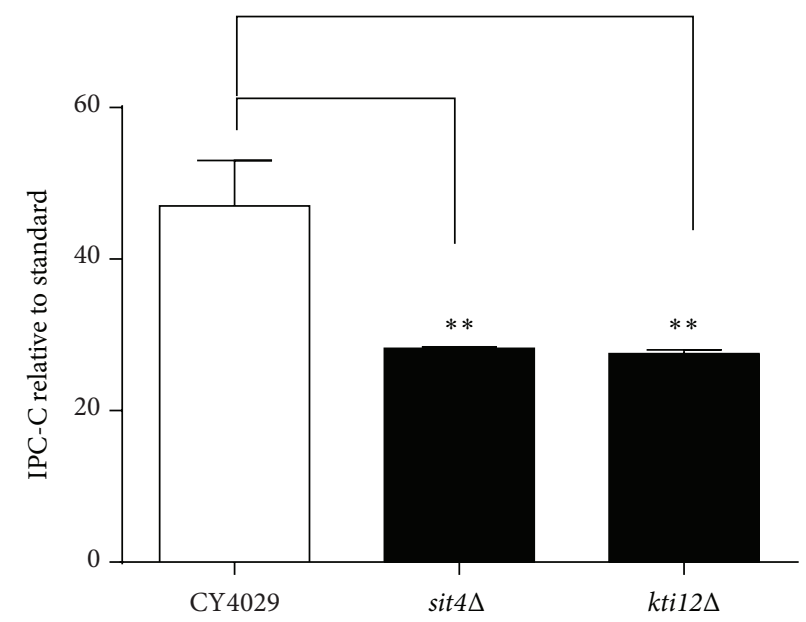

(c)

FIGURE 4: Mass spectrometric analysis of sphingolipid species. Yeast cultures were diluted to an $\mathrm{OD}_{600}$ of 0.2 in YPD and grown for 8 hours at $24^{\circ} \mathrm{C}$. A total of $10 \mathrm{OD}_{600}$ units of cells were removed and lipids extracted for mass spectrometry analysis. (a) Ceramides, (b) long chain bases phytosphingosine (PHS) and dihydrosphingosine (DHS), and (c) Inositol phosphoceramide-C (IPC-C) were quantified using relevant internal standards. Average values for a minimum of three biological replicates are shown and error bars represent the standard error above and below the mean. A Student's $t$-test was used to determine if the mutants showed a significant difference from the wild type CY4029. $\left({ }^{*} P<0.05,{ }^{* *} P<0.01\right.$, and $\left.{ }^{* * *} P<0.005\right)$.

(Figure 4(b)). However, a similar decrease in PHS levels in the kti12 $\Delta$ mutant does not correlate with resistance to exogenous PHS (Figures 4(b) and 3(c)), suggesting that a more complex mechanism underlies PHS resistance. There is a small increase in the levels of DHS in sit4 $\Delta$ and kti12 $\Delta$ mutants (Figure 4(b)) which is unlikely to affect the toxicity of DHS seen in Figure 3(b).

3.5. Sit4 Mutants Show an Increase in the Proportion of Dihydro Sphingolipids and a Corresponding Decrease in the Proportion of Hydroxylated Sphingolipids. Tritium labelled inositol incorporation was used to analyse the maturation of complex sphingolipid species formed from both dihydroceramide and phytoceramide. Dihydroceramide $\mathrm{B}^{\prime}$ $(18: 0 ; 2 / 26: 0 ; 0)$ and phytoceramide C (18:0;3/26:0;0) form inositol phosphoceramide B (IPC-B) and inositol phosphoceramide $\mathrm{C}$ (IPC-C) respectively. IPC-C and the corresponding MIPC-C generated from it form the relatively more abundant species of their sphingolipid class in the wild type (Figure 5). Interestingly, the sit $4 \Delta$ mutant contains increased levels of IPC-B and MIPC-B compared to the wild type, with a decrease in the levels of IPC-C and MIPC-C (Figure 5). The $k t i 12 \Delta$ mutant also shows a similar trend in the relative levels of sphingolipid species, but the differences from the wild type are less pronounced than those for sit4 $\Delta$. This indicates a shift towards more sphingolipids being synthesised from dihydroceramide/DHS precursors than from the hydroxylated phytoceramide/PHS precursors. Figure 4(c) provides additional evidence for this shift and quantification of IPCC levels shows a significant decrease in both sit $4 \Delta$ and ktil2 $\Delta$ mutants. This also correlates with the increase in DHS and 


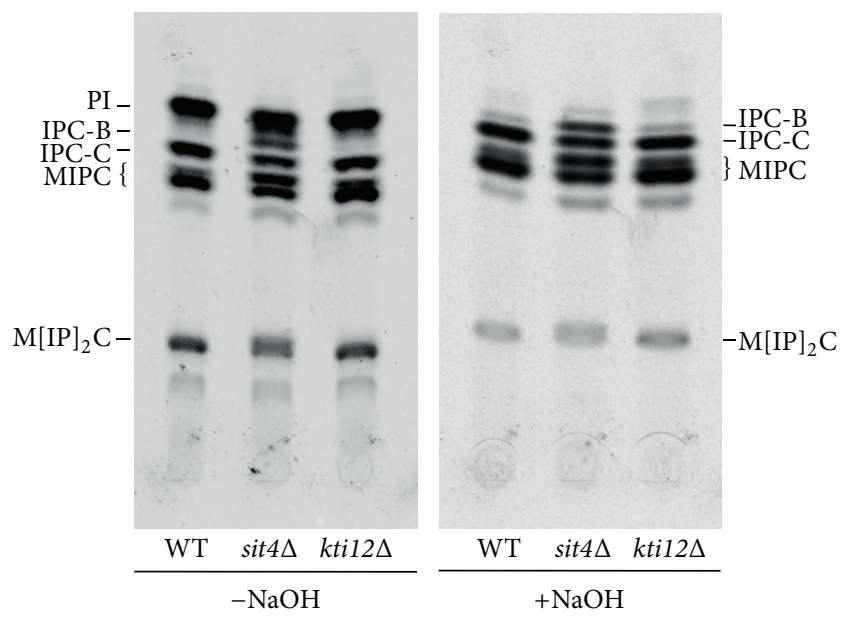

FIgURE 5: Tritium labelled inositol incorporation into yeast cells. CY4029 (WT), sit4 $\Delta$, and $k t i 12 \Delta$ were incubated with $\left[{ }^{3} \mathrm{H}\right]$-inositol for 4 hours, and lipids were extracted and analysed by thin layer chromatography, before and after mild-base treatment to remove inositol phosphate (PI). Equal CPMs were loaded for all the samples. A representative image of two biological replicates is shown.

decrease in PHS levels observed in sit4 $\Delta$ and $k t i 12 \Delta$ mutants (Figure 4(b)).

3.6. Novel Interactions of Sit $4 p$ with Tpd3p and Cdc55p Suggest a Role for an Alternative Phosphatase Complex in the Response to Ceramide. Previous studies suggested that Tpd $3 p$ and Cdc55p could be part of the CAPP complex as deletion of these genes conferred resistance to ceramide [10]. Indeed, we found via immunoprecipitation experiments with HA-labelled Sit $4 p$ that Tpd3p and Cdc55p-c-myc interact with Sit $4 \mathrm{p}$, forming a minor complex compared to the Pph21p/Tpd3p/Cdc55p complex (Figure 6). As this novel Sit4p/Tpd3p/Cdc55p trimer is formed constitutively and is not induced by the presence of ceramide (data not shown), the mechanism by which the phosphatase is activated in response to an increase in ceramide levels remains unclear.

\section{Discussion}

The aim of this study was to further investigate the role of the Sit4 phosphatase in response to ceramide and to determine if this signalling pathway is directly related to the biosynthesis of ceramides and sphingolipids. The phosphorylation status of Ormlp regulates the activity of serine palmitoyltransferase and therefore the production of all downstream products of the sphingolipid pathway. Ormlp is phosphorylated by Ypklp and evidence suggests that dephosphorylation may involve Sit4p and/or its TOR-dependent subunit Tap42p $[25,26]$. However, Ormlp is unlikely to be the direct substrate for the Sit4p or Tap42p phosphatases as mutation of these genes leads to decreased phosphorylation of Ormlp [25].

The Sit 4 phosphatase is a well-characterised regulator of tRNA modification via the Elongator complex [18, 21, 27-29].
However, here we show that the role of Sit $4 p$ in the ceramide response is independent of Elongator yet still involves the multifunctional and Elongator-related accessory protein Kti12p. Although previous work suggested that Elongator may be involved in ceramide resistance [18], more detailed analysis in this current study indicates that Elongator mutants are sensitive to ceramide. Although Ktil2p is essential for the phosphoregulation of Elongator, its precise role remains unclear $[18,21]$. Kti12p also has diverse roles in other cellular processes including the cell cycle [30] and transcription [31], and regulation of the ceramide response can now be added to this list.

The Sit 4 phosphatase has multiple regulatory subunits including the Sit 4 associated proteins (SAPs) Sap4p, Sap155p, Sap185p, and Sap190p. A quadruple deletion of all SAPs is sensitive to excess ceramide, in contrast to the resistant sit $4 \Delta$ mutant. Importantly, this is the first separation of function observed between the sit $4 \Delta$ and $\operatorname{sap} \Delta \Delta \Delta \Delta$ mutants. This suggests that an alternative Sit 4 phosphatase complex is involved in the regulation of the ceramide response, supporting the idea that this process is independent of Elongator functions that require Sit $4 /$ Sap complexes. The identification of a Sit $4 \mathrm{p} / \mathrm{Tpd} 3 \mathrm{p} / \mathrm{Cdc} 55 \mathrm{p}$ trimer also supports the theory that the ceramide activated protein phosphatase could be acting via a previously unknown mechanism.

The alteration of the sphingolipid makeup in sit4 $\Delta$ and $k t i 12 \Delta$ mutants and the decreased levels of ceramide and long chain bases indicate that there is regulation of the biosynthetic pathway at some level by Sit4p and/or Kti12p. The decreased level of endogenous ceramide and PHS in the mutants presumably enables them to survive an otherwise toxic concentration of these compounds. This suggests that deletion of SIT4 and KTI12 mediates a downregulation or partial inactivation of ceramide synthesis rather than a complete block, as there are clearly sufficient precursors available for effective biosynthesis of sphingolipids. The presence of multiple genes encoding enzymes for synthesis and degradation of ceramides is a key way in which sphingolipid metabolism can be maintained when the pathway is partially blocked. Phosphoregulation of ceramide synthases has not been previously observed, but three phosphorylated serine residues are conserved in both Laglp and Laclp ceramide synthases and could be potential targets for dephosphorylation by Sit4p [32,33]. In common with sit4 $\Delta$ and ktil2 $\Delta$ mutants, lac1 $\Delta$ lag1 $\Delta$ mutants are resistant to the tRNase toxin zymocin, but the mechanism of action is due to a defect in plasma membrane integrity caused by decreased levels of the sphingolipid $\mathrm{M}(\mathrm{IP})_{2} \mathrm{C}$ and not via Elongator [34].

In sit $4 \Delta$ and ktil2 $\Delta$ mutants, the relative proportion of lipids synthesised from dihydroceramides/DHS is higher than those synthesised from phytoceramides/PHS, suggesting that there could be a defect in the Sur2 hydroxylase which hydroxylates both long chain bases and ceramides [35]. This is also reflected in the increased levels of DHS seen in the mutants and is therefore unlikely to simply be a defect in the synthesis of ceramide or downregulation at an earlier stage in the pathway, as not all components of the pathway are downregulated. The ceramidases Ypclp and Ydclp also have a minor ceramide synthase activity and show specificity for 


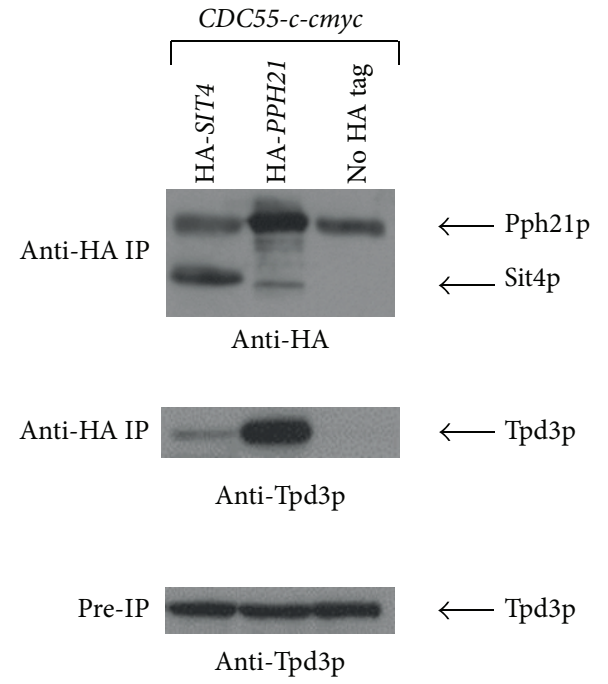

(a)
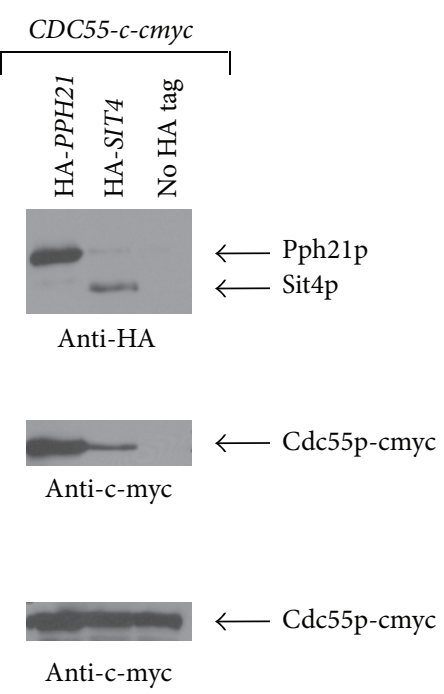

(b)

FIGURE 6: Immunoprecipitation of HA-Sit4p reveals novel interactors. Equal amounts of protein extracts were immunoprecipitated with magnetic beads coated with anti-HA antibodies, and the precipitates were then subjected to Western blotting and probed with anti-HA and anti-Tpd3 (a) or anti-c-myc (b) antibodies. Equal amounts of total protein extracts (without immunoprecipitation) were also probed with anti-Tpd3 or anti-c-myc antibodies.

hydroxylated and nonhydroxylated forms of long chain bases, respectively. [36] Dysregulation in sit4 $\Delta$ could cause a shift towards the synthesis of nonhydroxylated sphingolipids by these enzymes. However, these enzymes contribute a minor level of ceramide synthase activity compared to Laglp, Laclp, and Liplp [37], so a change in their regulation is unlikely to have any detrimental effects on the overall sphingolipid composition of the plasma membrane, even if the balance of individual components is altered.

These new insights into the novel ceramide-associated functions of Sit $4 p$ and Ktil2 $p$ are helpful in understanding the diverse roles these proteins play in the cell and expand our knowledge of their importance beyond their association with the Elongator complex. Relatively little is known about the human orthologues of Sit $4 p$ and Ktil2p, and thus yeast studies are vital in unravelling the essential role they play in regulating cell proliferation and cell death in both healthy and malignant cells.

\section{Conclusions}

This study indicates that the roles of Sit $4 p$ and Ktil2p in the ceramide response are distinct from their roles in the regulation of the Elongator complex and are therefore likely to be mediated via a novel mechanism. The separation of function between sit4 $\Delta$ and $\operatorname{sap} \Delta \Delta \Delta \Delta$ mutants and the interaction of Sit $4 p$ with the alternative regulatory subunits Cdc55p and Tpd3p also support this theory. Alterations in the levels of ceramides, long chain bases, and complex sphingolipids in sit $4 \Delta$ and ktil2 $\Delta$ mutants indicate that these proteins are also likely to regulate the sphingolipid biosynthesis pathway. Future work will be targeted at delineating the underlying mechanism(s) underlying these observations.

\section{Acknowledgments}

This work was funded by The Wellcome Trust (Grant no. WT088104MA). The authors would like to thank Yu Jiang for the donation of anti-Tpd3p antibody and Michael Stark and Robert Mason for helpful discussions.

\section{References}

[1] G. M. Jenkins and Y. A. Hannun, "Role for de Novo sphingoid base biosynthesis in the heat-induced transient cell cycle arrest of Saccharomyces cerevisiae," Journal of Biological Chemistry, vol. 276, no. 11, pp. 8574-8581, 2001.

[2] T. Kolter, "A view on sphingolipids and disease," Chemistry and Physics of Lipids, vol. 164, no. 6, pp. 590-606, 2011.

[3] P. P. Ruvolo, "Intracellular signal transduction pathways activated by ceramide and its metabolites," Pharmacological Research, vol. 47, no. 5, pp. 383-392, 2003.

[4] S. A. Saddoughi, P. Song, and B. Ogretmen, "Roles of bioactive sphingolipids in cancer biology and therapeutics," Sub-Cellular Biochemistry, vol. 49, pp. 413-440, 2008.

[5] S. Gatt and A. Dagan, "Cancer and sphingolipid storage disease therapy using novel synthetic analogs of sphingolipids," Chemistry and Physics of Lipids, vol. 165, no. 4, pp. 462-474, 2012.

[6] S. Ponnusamy, M. Meyers-Needham, C. E. Senkal et al., "Sphingolipids and cancer: ceramide and sphingosine-1-phosphate in the regulation of cell death and drug resistance," Future Oncology, vol. 6, no. 10, pp. 1603-1624, 2010.

[7] L. K. Ryland, T. E. Fox, X. Liu, T. P. Loughran, and M. Kester, "Dysregulation of sphingolipid metabolism in cancer," Cancer Biology and Therapy, vol. 11, no. 2, pp. 138-149, 2011.

[8] R. C. Dickson, "New insights into sphingolipid metabolism and function in budding yeast," Journal of Lipid Research, vol. 49, no. 5, pp. 909-921, 2008. 
[9] N. Bartke and Y. A. Hannun, "Bioactive sphingolipids: metabolism and function," Journal of Lipid Research, vol. 50, supplement, pp. S91-96, 2009.

[10] J. T. Nickels and J. R. Broach, "A ceramide-activated protein phosphatase mediates ceramide-induced G1 arrest of Saccharomyces cerevisiae," Genes and Development, vol. 10, no. 4, pp. 382-394, 1996.

[11] M. A. de la Torre-Ruiz, J. Torres, J. Ariño, and E. Herrero, "Sit4 is required for proper modulation of the biological functions mediated by $\mathrm{Pkcl}$ and the cell integrity pathway in Saccharomyces cerevisiae," Journal of Biological Chemistry, vol. 277, no. 36, pp. 33468-33476, 2002.

[12] A. R. Butler, R. W. O’Donnell, V. J. Martin, G. W. Gooday, and M. J. R. Stark, "Kluyveromyces lactis toxin has an essential chitinase activity," European Journal of Biochemistry, vol. 199, no. 2, pp. 483-488, 1991.

[13] B. Huang, J. Lu, and A. S. Byström, "A genome-wide screen identifies genes required for formation of the wobble nucleoside 5-methoxycarbonylmethyl-2-thiouridine in Saccharomyces cerevisiae," RNA, vol. 14, no. 10, pp. 2183-2194, 2008.

[14] Y. Jiang and J. R. Broach, "Tor proteins and protein phosphatase 2 A reciprocally regulate Tap42 in controlling cell growth in yeast," EMBO Journal, vol. 18, no. 10, pp. 2782-2792, 1999.

[15] M. N. Miranda, C. A. Masuda, A. Ferreira-Pereira, E. Carvajal, M. Ghislain, and M. Montero-Lomelí, "The serine/threonine protein phosphatase Sit4p activates multidrug resistance in Saccharomyces cerevisiae," FEMS Yeast Research, vol. 10, no. 6, pp. 674-686, 2010.

[16] A. Sutton, D. Immanuel, and K. T. Arndt, "The SIT4 protein phosphatase functions in late G1 for progression into S phase," Molecular and Cellular Biology, vol. 11, no. 4, pp. 2133-2148, 1991.

[17] C. J. Di Como and K. T. Arndt, "Nutrients, via the Tor proteins, stimulate the association of Tap42 with type 2A phosphatases," Genes and Development, vol. 10, no. 15, pp. 1904-1916, 1996.

[18] D. Jablonowski, L. Fichtner, M. J. R. Stark, and R. Schaffrath, "The yeast elongator histone acetylase requires Sit4-dependent dephosphorylation for toxin-target capacity," Molecular Biology of the Cell, vol. 15, no. 3, pp. 1459-1469, 2004.

[19] D. Jablonowski, J.-E. Täubert, C. Bär, M. J. R. Stark, and R. Schaffrath, "Distinct subsets of Sit4 holophosphatases are required for inhibition of Saccharomyces cerevisiae growth by rapamycin and zymocin," Eukaryotic Cell, vol. 8, no. 11, pp. 1637$1647,2009$.

[20] M. M. Luke, F. D. Seta, C. J. Di Como, H. Sugimoto, R. Kobayashi, and K. T. Arndt, "The SAPs, a new family of proteins, associate and function positively with the SIT4 phosphatase," Molecular and Cellular Biology, vol. 16, no. 6, pp. 2744-2755, 1996.

[21] C. Mehlgarten, D. Jablonowski, K. D. Breunig, M. J. R. Stark, and R. Schaffrath, "Elongator function depends on antagonistic regulation by casein kinase Hrr25 and protein phosphatase Sit4," Molecular Microbiology, vol. 73, no. 5, pp. 869-881, 2009.

[22] D. Jablonowski, F. Frohloff, L. Fichtner, M. J. R. Stark, and R. Schaffrath, "Kluyveromyces lactis zymocin mode of action is linked to RNA polymerase II function via Elongator," Molecular Microbiology, vol. 42, no. 4, pp. 1095-1105, 2001.

[23] S. Han, M. A. Lone, R. Schneiter, and A. Chang, "Orml and Orm2 are conserved endoplasmic reticulum membrane proteins regulating lipid homeostasis and protein quality control," Proceedings of the National Academy of Sciences of the United States of America, vol. 107, no. 13, pp. 5851-5856, 2010.
[24] F. Reggiori, E. Canivenc-Gansel, and A. Conzelmann, "Lipid remodeling leads to the introduction and exchange of defined ceramides on GPI proteins in the ER and Golgi of Saccharomyces cerevisiae," EMBO Journal, vol. 16, no. 12, pp. 3506-3518, 1997.

[25] M. Liu, C. Huang, S. R. Polu, R. Schneiter, and A. Chang, "Regulation of sphingolipid synthesis through Orm1 and Orm2 in yeast," Journal of Cell Science, vol. 125, no. 10, pp. 2428-2435, 2012.

[26] F. M. Roelants, D. K. Breslow, A. Muir, J. S. Weissman, and J. Thorner, "Protein kinase Ypk1 phosphorylates regulatory proteins Orm1 and Orm2 to control sphingolipid homeostasis in Saccharomyces cerevisiae," Proceedings of the National Academy of Sciences of the United States of America, vol. 108, no. 48, pp. 19222-19227, 2011.

[27] B. Huang, M. J. O. Johansson, and A. S. Byström, "An early step in wobble uridine tRNA modification requires the Elongator complex," RNA, vol. 11, no. 4, pp. 424-436, 2005.

[28] D. Jablonowski, S. Zink, C. Mehlgarten, G. Daum, and R. Schaffrath, "tRNAGlu wobble uridine methylation by Trm9 identifies Elongator's key role for zymocin-induced cell death in yeast," Molecular Microbiology, vol. 59, no. 2, pp. 677-688, 2006.

[29] C. Mehlgarten, D. Jablonowski, U. Wrackmeyer et al., "Elongator function in tRNA wobble uridine modification is conserved between yeast and plants," Molecular Microbiology, vol. 76, no. 5, pp. 1082-1094, 2010.

[30] A. R. Butler, J. H. White, Y. Folawiyo, A. Edlin, D. Gardiner, and M. J. R. Stark, "Two Saccharomyces cerevisiae genes which control sensitivity to G1 arrest induced by Kluyveromyces lactis toxin," Molecular and Cellular Biology, vol. 14, no. 9, pp. 63066316, 1994.

[31] T. G. Petrakis, T. M. M. Søgaard, H. Erdjument-Bromage, P. Tempst, and J. Q. Svejstrup, "Physical and functional interaction between elongator and the chromatin-associated Ktil2 protein," Journal of Biological Chemistry, vol. 280, no. 20, pp. 1945419460, 2005.

[32] B. Bodenmiller, D. Campbell, B. Gerrits et al., "PhosphoPep: a database of protein phosphorylation sites in model organisms," Nature Biotechnology, vol. 26, no. 12, pp. 1339-1340, 2008.

[33] A. Huber, B. Bodenmiller, A. Uotila et al., "Characterization of the rapamycin-sensitive phosphoproteome reveals that Sch9 is a central coordinator of protein synthesis," Genes and Development, vol. 23, no. 16, pp. 1929-1943, 2009.

[34] S. Zink, C. Mehlgarten, H. K. Kitamoto et al., "Mannosyldiinositolphospho-ceramide, the major yeast plasma membrane sphingolipid, governs toxicity of Kluyveromyces lactis zymocin," Eukaryotic Cell, vol. 4, no. 5, pp. 879-889, 2005.

[35] M. M. Grilley, S. D. Stock, R. C. Dickson, R. L. Lester, and J. Y. Takemoto, "Syringomycin action gene SYR2 is essential for sphingolipid 4- hydroxylation in Saccharomyces cerevisiae," Journal of Biological Chemistry, vol. 273, no. 18, pp. 11062-11068, 1998.

[36] C. Mao, R. Xu, A. Bielawska, and L. M. Obeid, "Cloning of an alkaline ceramidase from Saccharomyces cerevisiae. An enzyme with reverse (CoA-independent) ceramide synthase activity," Journal of Biological Chemistry, vol. 275, no. 10, pp. 6876-6884, 2000.

[37] C. Mao, R. Xu, A. Bielawska, Z. M. Szulc, and L. M. Obeid, "Cloning and characterization of a Saccharomyces cerevisiae alkaline ceramidase with specificity for dihydroceramide," Journal of Biological Chemistry, vol. 275, no. 40, pp. 31369-31378, 2000 . 


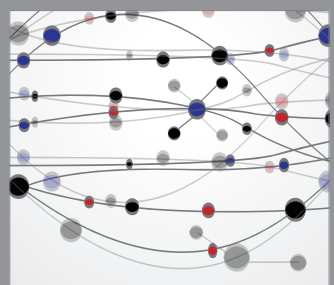

The Scientific World Journal
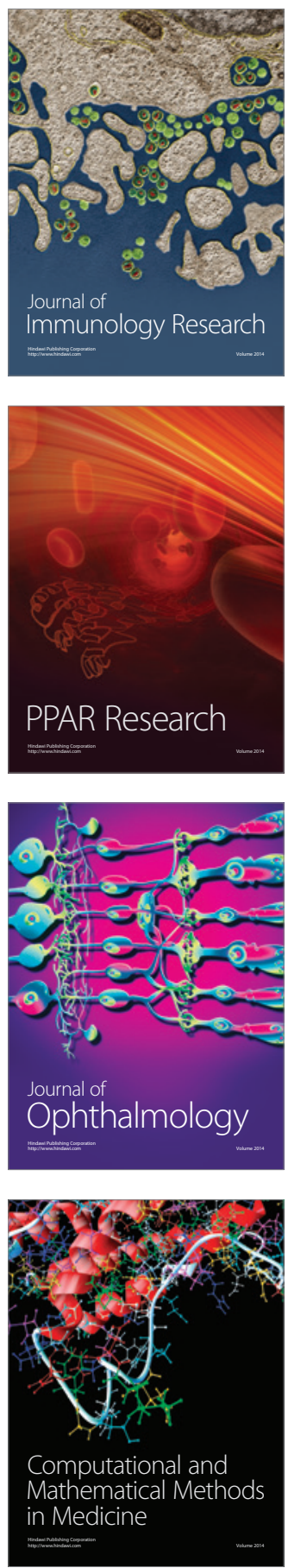

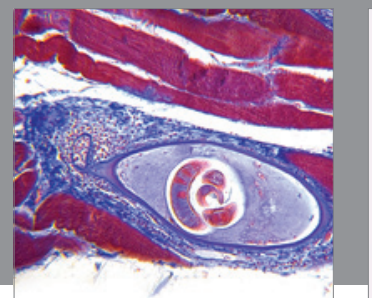

Gastroenterology

Research and Practice
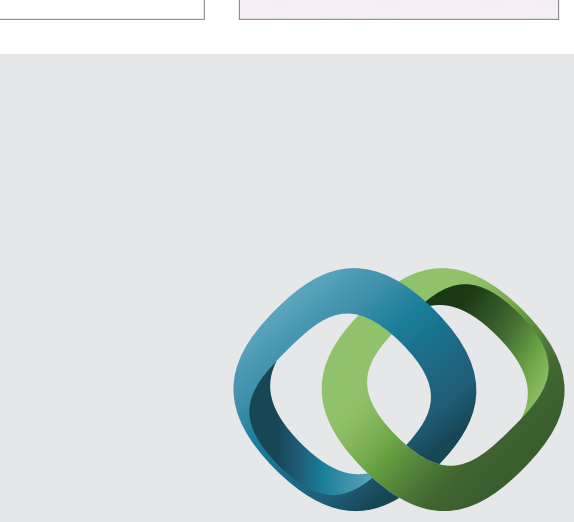

\section{Hindawi}

Submit your manuscripts at

http://www.hindawi.com
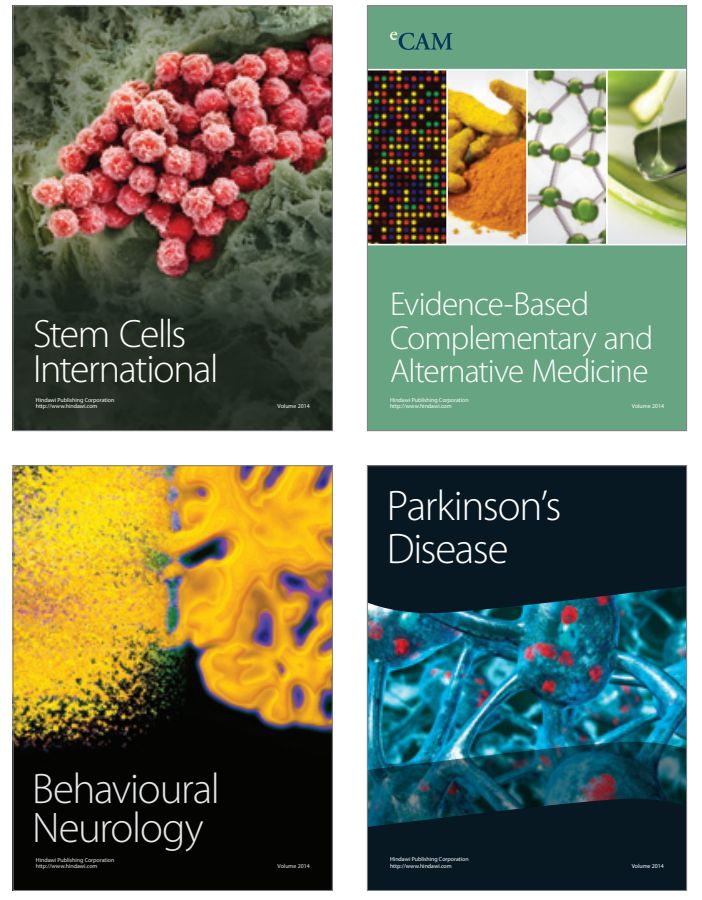
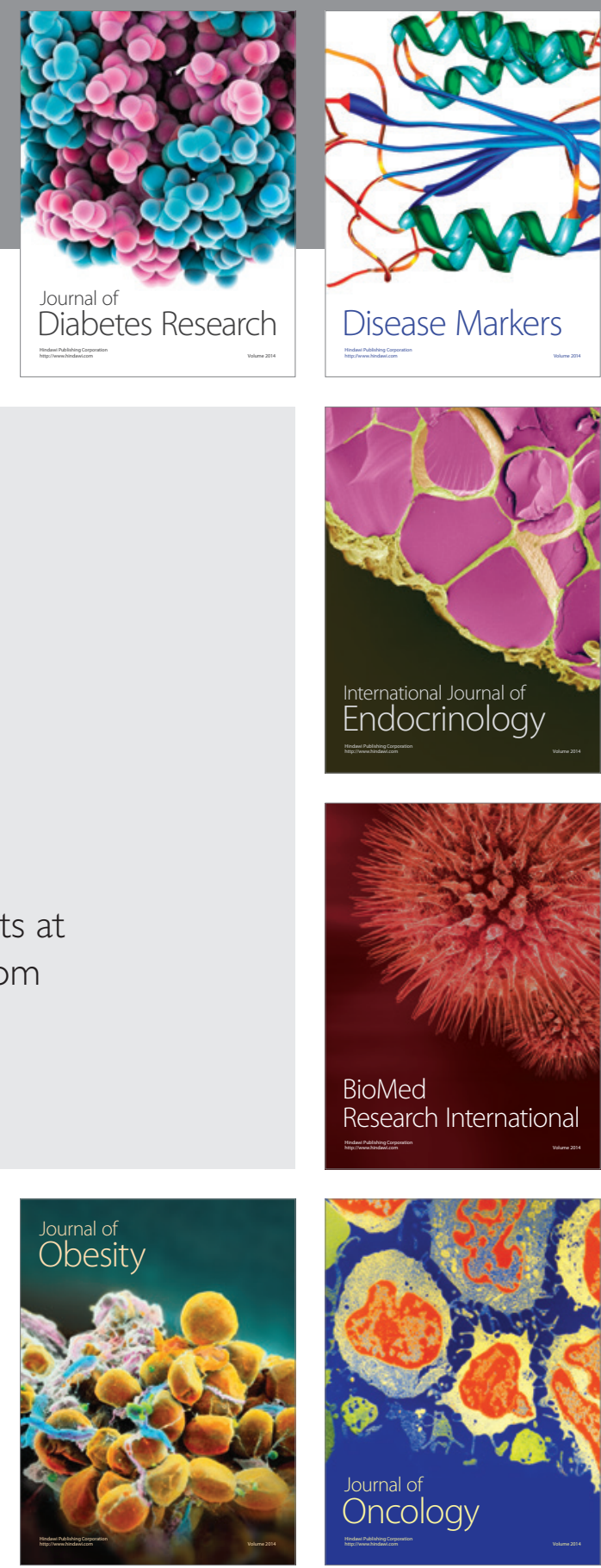

Disease Markers
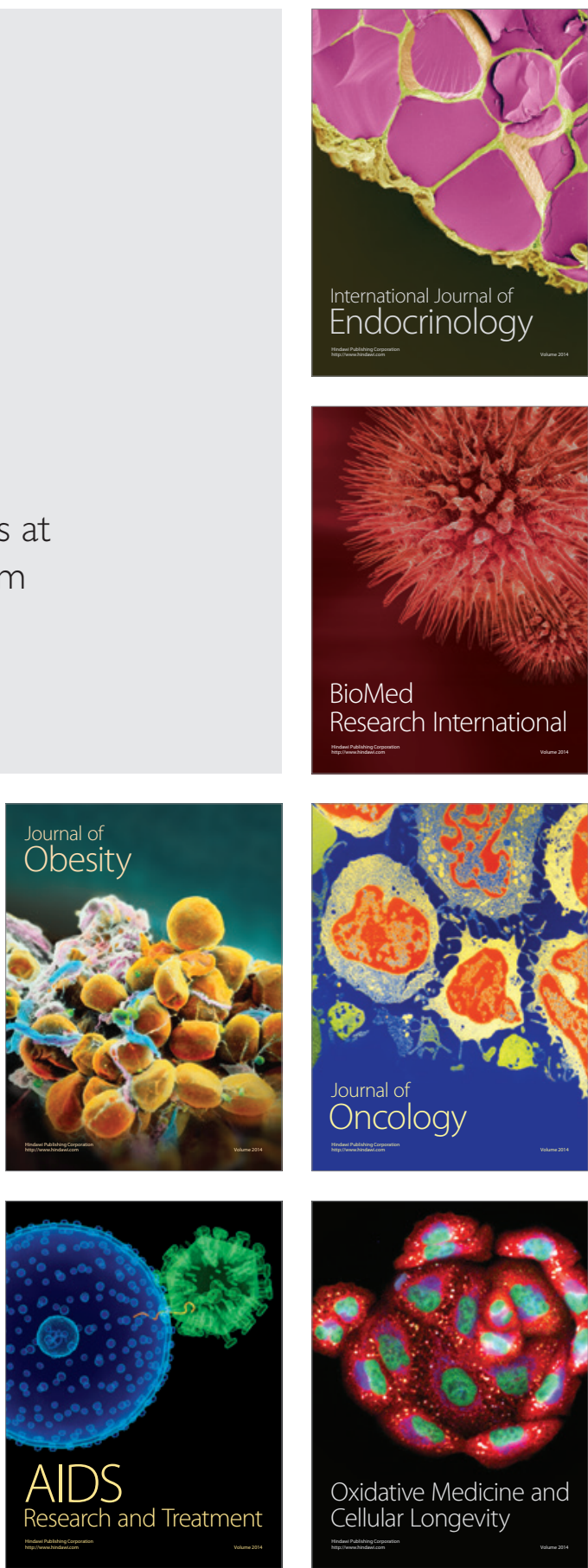